\title{
Ciclos cortos de Educación Superior en Europa: variabilidad institucional y dimensión social
}

\author{
Savall Ceres, Juana \\ Universidad Francisco de Vitoria, Madrid, España \\ j.savall@ufv.es
}

\section{Resumen}

La denominada educación terciaria de ciclo corto, que en el sistema educativo español corresponde con la Formación Profesional de Grado Superior, ha ido ganado terreno en Europa y se ha integrado en los marcos de cualificaciones de Educación Superior en muchos países. No obstante, sigue siendo un sector educativo generalmente desconocido, institucionalmente heterogéneo y con desigual valoración social en el entorno supranacional.

En la presente comunicación se profundiza en su origen y situación actual. Hoy en día, la ciudadanía activa y el compromiso social son cuestiones de especial interés para muchas de las instituciones que ofrecen Educación Superior de ciclo corto en toda Europa, dada la diversidad de su alumnado y su papel en el aprendizaje permanente.

Como ya se señaló en la Conferencia Mundial sobre la Educación Superior (UNESCO 2009) «en ningún otro momento de la historia ha sido más importante que ahora la inversión en los estudios superiores, por su condición de fuerza primordial para la construcción de sociedades del conocimiento integradoras y diversas, y para fomentar la investigación, la innovación y la creatividad. [...] La sociedad del conocimiento necesita diversos sistemas de Educación Superior, con una gama de instituciones que tengan cometidos variados y lleguen a diversos tipos de educandos».

\section{Abstract}

The named short-cycle tertiary education which corresponds to the "Formación Profesional de Grado Superior" in the spanish educational system, has gained ground in Europe and it has been integrated into the qualification frameworks of Higher Education in many countries. Nevertheless, it is still an educational sector generally unknown, institutionally heterogeneous and with unequal social assessments within the supranational context.

In the present notification its origin and actual situation are examined in depth. The active citizenship and the social commitment are issues of special interest for many of the institutions which provide short-cycle Higher Education in all Europe, because of their student diversity and their role in permanent learning.

As it was stated in the World Conference about Higher Education (UNESCO 2009) "at no time in history has it been more important to invest in higher education as a major force in building an inclusive and diverse knowledge society and to advance research, innovation and creativity. [...] The knowledge society needs diversity in higher education systems, with a range of institutions having a variety of mandates and addressing different types of learners".

Palabras clave: Educación Superior, ciclos cortos, Formación Profesional, universidad, instituciones.

Keywords: higher education, short cycles, vocational training, university, institutions.

\section{INTRODUCCIÓN}

Hablar acerca de los llamados «ciclos cortos» de la Educación Superior no es algo nuevo. Ya en 1973, la Organización para la Cooperación y el Desarrollo Económico (OCDE) los caracterizó como «[...] la educación post-secundaria de menor duración con elementos vocacionales fuertes, generalmente bajo el sector no universitario de la enseñanza superior [...]». No obstante, su definición se encuentra todavía en la vanguardia de los debates políticos en muchos países (Cremonini 2010). 
En esta comunicación se analizará el origen de este sector de la Educación Superior y su configuración en el ámbito europeo, destacando la diversidad de instituciones en las que se imparte y el heterogéneo perfil de sus estudiantes para, finalmente, destacar su dimensión social y valor en la cadena del aprendizaje permanente.

\section{ORIGEN DE LOS CICLOS CORTOS DE EDUCACIÓN SUPERIOR}

En las últimas décadas, la Educación Superior se ha diversificado institucionalmente, básicamente en dos dimensiones (Jacinto 2013). En sentido horizontal, se ha incrementado el número de universidades públicas y privadas que ofertan, asimismo, un mayor número de títulos universitarios. Esta diversificación horizontal de la oferta es aún mayor si se tiene en cuenta la expansión en los últimos años de la educación a distancia. En sentido vertical, los sistemas se diferencian habitualmente entre instituciones y programas universitarios e instituciones y programas no universitarios.

Como subraya Teichler (2009), a partir de los años sesenta apareció en Europa un nuevo sector de la Educación Superior, aunque dicha aparición se produjo en diferentes momentos en cada país. Los primeros modelos, es decir, las «politécnicas» británicas, los Instituts Universitaires de Technologie (IUT) franceses y la Fachhochschulen alemana, presentaban diferencias en no pocos sentidos, aunque en general compartían una serie de tendencias.

En primer lugar, González Silva (2006) afirma que su surgimiento constituye una respuesta de los Estados ante la incapacidad de los modelos universitarios existentes para recibir la creciente demanda de estudios superiores. Esta tendencia se cruza con las necesidades del sector empresarial y su demanda de funcionalidad de la Educación Superior con respecto a su adaptación a las condiciones del mercado de trabajo. Junto a las dos primeras tendencias, aparece la necesidad de revisión de los modelos universitarios en busca de flexibilidad y adaptación de las ofertas profesionales y de la organización para atenderlas, de un mayor control de la Educación Superior por parte de los gobiernos y de tender un puente al sector privado para generar un crecimiento que no supusiese las inversiones que el modelo universitario (con exigencia de profesionalización y carrera docente, instalaciones para la docencia y la investigación, etc.) requiere.

De esta forma, aunque las necesidades y voluntades se encaminaban hacia una misma dirección, las respuestas institucionales no fueron las mismas en esta diversificación de la Educación Superior. Se propusieron diferentes intentos para designar a esta esfera de la Educación Superior (Teichler 2009), mas nunca se alcanzó un consenso en cuanto al término más adecuado:

- Educación Superior de primer ciclo

- Educación Superior no universitaria

— «Sector alternativo» de la Educación Superior

- Educación superior técnica

- Educación superior profesional

- Etc.

No existe pues una denominación única ni un concepto unívoco. Más bien ante un campo difuso, con límites indefinidos, constituido por distintos tipos de instituciones.

\section{TENDENCIAS EUROPEAS}

La organización de los denominados programas de Educación Superior de ciclo corto es heterogénea en los distintos países europeos. Dichos programas generalmente están diseñados para ofrecer a los estudiantes conocimientos, destrezas y competencias profesionales. Suelen tener una base práctica centrada en una ocupación específica para preparar a los estudiantes para acceder al mercado de trabajo. Sin embargo, también suelen dar paso a otros programas de Educación Superior. 


\subsection{Variabilidad de los programas}

En un informe de la red Eurydice (2013) se subraya que tanto la tipología como el número de instituciones de Educación Superior presentan diferencias en los países del Espacio Europeo de la Educación Superior (EEES). Los centros de Educación Superior pueden variar en cuanto a su orientación, académica o profesional, ser de titularidad pública o privada, sostenerse con fondos públicos o privados, y presentar otro tipo de diferencias según el contexto específico de cada país.

En primer lugar, las instituciones de Educación Superior pueden tener una orientación académica o profesional. Sin embargo, la diferencia entre estos dos modelos es cada vez menos nítida. En muchos países persiste la antigua diferenciación formal entre ambos tipos de instituciones, pero en la práctica -en parte como consecuencia del Proceso de Bolonia- esta distinción está diluyéndose, o ya ha desaparecido por completo.

En el Comunicado de Berlín de 2003 los ministros instaron a que se estudiara más a fondo «la posibilidad y las vías para vincular la Educación Superior de ciclo corto con el primer ciclo del Marco de Cualificaciones para el EEES». Los programas de corta duración se introdujeron dentro del Marco de Cualificaciones del EEES mediante la incorporación al mismo de una oferta adicional de ciclo corto o vinculándolos al primer ciclo.

El documento del Marco Europeo de Cualificaciones (MEC) asegura la compatibilidad de los niveles superiores del MEC con el Marco de Cualificaciones del Espacio Europeo de Educación Superior (MC-EEES), pero, aunque los resultados del aprendizaje de determinados niveles del MEC se corresponden con los descriptores de ciclo del MC-EEES y existen claras referencias cruzadas, no todos los países que han desarrollado programas de formación profesional superior en el nivel 5 del MEC los consideran equivalentes a la Educación Superior de ciclo corto.

No hay que olvidar que, por un lado, ambos marcos presentan ciertas similitudes pero responden a fines diferentes. El objetivo principal del MC-EEES es armonizar los sistemas de Educación Superior de Europa mediante la introducción de estructuras de titulación comunes con un número aproximado de créditos ECTS, aumentando así la transparencia, el reconocimiento y la movilidad, mientras que el MEC es un marco global que está mucho más orientado al mercado laboral.

En este sentido, en un reciente estudio del CEDEFOP (2014) se identifican 31 tipos de cualificaciones vinculados al nivel 5 del Marco Europeo de Cualificaciones en los 15 países analizados.

Mientras que los anteriores estudios se habían centrado en un solo tipo de cualificaciones de nivel 5 , las cualificaciones de la Educación Superior de ciclo corto, este estudio de CEDEFOP abarca todo tipo de cualificaciones de nivel 5 de los distintos subsistemas.

Como se demuestra en este estudio, el nivel 5 del Marco Europeo de Cualificaciones (MEC) abarca la formación profesional, la Educación Superior y la educación general, e incluye una variedad muy heterogénea de cualificaciones concedidas por un amplio abanico de instituciones de formación profesional y Educación Superior. La mitad de las cualificaciones de nivel 5 están reguladas por el sistema de Educación Superior o son impartidas por instituciones de Educación Superior (Bélgica-Flandes, Dinamarca, Irlanda, Francia, Croacia, Letonia, Luxemburgo, Malta, Países Bajos y Reino Unido).

Si se tienen en cuenta los resultados del aprendizaje de estas cualificaciones, la distinción entre la formación profesional y la Educación Superior puede resultar poco clara. Los vínculos entre las instituciones y los distintos tipos de cualificaciones también se van debilitando. En muchos casos, el modo de adquisición de las cualificaciones de nivel 5 también es muy flexible. Por ejemplo, el Diploma Universitario de Tecnología en Gestión de Empresas y Administraciones (DUT-GEA) francés, basado en el enfoque de los resultados del aprendizaje, se puede obtener por vías muy distintas: principalmente a través de los institutos universitarios de tecnología (IUT), formación profesional continua para los estudiantes que trabajan a través de, por ejemplo, el contrato de profesionalización (CP); a través de la validación de los aprendizajes adquiridos previamente (VAE)... 


\subsubsection{Introducción de la Educación Superior de ciclo corto}

Centrándose en los ciclos cortos de Educación Superior, el objetivo general de un estudio comparativo, publicado en 2013 por la Asociación Europea de Instituciones de Educación Superior (EURAS$\mathrm{HE}$ ), fue hacer un análisis detallado de ellos en 32 de los países signatarios de Bolonia. Su título resulta muy ilustrativo: Short Cycle Higher Education in Europe Level 5: the Missing Link.

Se pueden distinguir tres grupos de países teniendo en cuenta las fechas en las que los distintos países han introducido la Educación Superior de ciclo corto. En primer lugar, Francia, los países de habla inglesa (Irlanda, Reino Unido), Noruega y Chipre introdujeron la Educación Superior de ciclo corto en los años sesenta o setenta. Un segundo grupo de países, formado principalmente por los países de Europa Central y Oriental (Bulgaria, República Checa, Hungría, Eslovenia), aunque también por Islandia (1990) y Letonia (2001), la introdujo como educación postsecundaria superior en los años noventa o principios del segundo milenio. Finalmente están los países que la introdujeron tras la adopción del MC-EEES y, en algunos casos, después de haber introducido/construido su propio marco nacional de cualificaciones (Bélgica-Comunidad flamenca, Bélgica-Comunidad francesa, Dinamarca, Malta, Países Bajos). En Bélgica y en los Países Bajos la introducción de la Educación Superior de ciclo corto fue un proceso de transformación de la formación profesional superior más que de introducción de algo completamente nuevo.

\subsubsection{Oferta institucional}

Una de las características de la Educación Superior de ciclo corto es que se ofrece en una amplia variedad de escenarios, no sólo en Europa sino en todos y cada uno de los países del estudio de EURASHE. Solo hay tres países donde la ofrece un único tipo de institución. En Islandia solamente las universidades ofrecen Educación Superior de ciclo corto y estas deben haber sido previamente acreditadas por el gobierno. En el caso de los Países Bajos, puede ser ofrecida en diferentes escenarios, aunque siempre se trata de universidades de ciencias aplicadas. En Turquía las universidades y las universidades pertenecientes a fundaciones son las únicas que ofrecen este tipo de educación, aunque puede ser organizada por escuelas administradas por fundaciones.

No obstante, en la mayor parte de los países del estudio (Bélgica-Comunidad flamenca, Francia, Hungría, Irlanda, Islandia, Letonia, Luxemburgo, Malta, Países Bajos, Noruega, Portugal, Eslovenia, Turquía, Reino Unido-Inglaterra/Gales/Irlanda del Norte, Reino Unido-Escocia), la Educación Superior de ciclo corto es ofrecida por instituciones de Educación Superior como universidades, universidades de ciencias aplicadas, institutos técnicos regionales o escuelas universitarias. La comunidad francesa de Bélgica también tiene la intención de organizar este tipo de educación en escuelas universitarias en un futuro. En Chipre, la República Checa, Dinamarca, España, Letonia, Malta, Eslovenia, Inglaterra, Gales e Irlanda del Norte, las escuelas profesionales o técnicas (también) ofrecen Educación Superior de ciclo corto, y en Chipre, Dinamarca, Irlanda y el Reino Unido también los centros de educación postobligatoria. En Flandes, la República Checa, Francia, Hungría, España y Luxemburgo, los centros de educación secundaria superior también pueden ofrecerla. En el caso de Flandes, esto ocurre únicamente en un programa (Enfermería) y, en la República Checa, suele tratarse de instituciones distintas dentro de un mismo centro de secundaria. En Portugal, los cursos de especialización tecnológica (CET, o cursos de Educación Superior de ciclo corto) los pueden ofrecer las instituciones de Educación Superior, la red de escuelas estatales, privadas y cooperativas, las escuelas profesionales, así como los centros gestionados total o parcialmente por el Instituto de Empleo y Formación Profesional (IEFP). Por último, en Bélgica (comunidades flamenca y francesa), la Educación Superior de ciclo corto se ofrece en el marco de la educación de adultos, en los denominados centros de educación de adultos. Conviene señalar que, aunque en el pasado las instituciones belgas de Educación Superior apenas se centraban en los estudiantes adultos, el país posee una floreciente tradición de educación de adultos para la promoción social. Aunque en otros países también se atiende a este tipo de estudiantes, aquí los cursos para estudiantes adultos tienen lugar en diversos escenarios, como centros de educación postobligatoria, escuelas profesionales, universidades y escuelas universitarias. (pp. 39-40). 


\subsubsection{Duración de los estudios}

A todos los encuestados del estudio de EURASHE se les preguntó si la duración de la Educación Superior de ciclo corto se expresaba en años o en créditos ECTS y cuántos años (a tiempo completo) o créditos ECTS comprendían los estudios.

La mayoría de los países todavía expresa la duración de este tipo de educación en años. En ocho países (Bélgica-Comunidad francesa, España, Francia, Hungría, Luxemburgo, Noruega, Turquía, Reino Unido-Inglaterra/Gales/Irlanda del Norte) los estudios (a tiempo completo) tienen una duración de dos años. En dos países (Islandia, Portugal) estos duran entre uno y dos años. En Chipre, la duración de los estudios depende del título que los estudiantes quieran obtener. En la República Checa todavía hay cursos de tres años (incluso tres años y medio en el caso de enfermería), aunque es posible que estos estudios se conviertan en un Grado profesional en un futuro.

El resto de países expresa la duración en créditos (ECTS). En tres países (Irlanda, Países Bajos, Eslovenia), los estudiantes tienen que obtener 120 créditos ECTS. En los Países Bajos, el mínimo requerido es de 120 créditos ECTS, aunque en la práctica todos los programas tienen 120 créditos ECTS. En tres países, los estudiantes deben obtener entre 90 y 120 créditos ECTS (Bélgica-Comunidad flamenca, Malta, Reino Unido-Escocia). En el caso de la comunidad flamenca de Bélgica, el número de créditos ECTS necesarios es de 90 o 120. Portugal expresa la duración de este tipo de estudios en créditos ECTS (80), años (tres semestres) y horas (840-

1.200 horas, sin incluir la formación en centros de trabajo). Finalmente, Letonia indicó que estos estudios equivalen a 80-120 créditos letones (un crédito letón solía ser 1,5 veces mayor que un crédito ECTS) y en Dinamarca es necesario obtener entre 90 y 150 créditos ECTS.

\subsection{Dimensión social}

Siguiendo con el estudio de EURASHE, aunque en prácticamente todos los países la mayoría de los estudiantes estudia a tiempo completo, en varios países existe una mayoría (Eslovenia 55\%) o un importante porcentaje que lo hace a tiempo parcial (Letonia 48\%, Países Bajos 45\%, Reino Unido-Inglaterra/Gales/Irlanda del Norte $43 \%$ e Irlanda $43 \%$ ).

Aunque en los Países Bajos, Reino Unido-Inglaterra/Gales/Irlanda del Norte y Dinamarca la mayoría de los estudiantes estudia a tiempo completo, existe una mayoría de estudiantes adultos. En los Países Bajos el $70 \%$ de los estudiantes de Educación Superior de ciclo corto son estudiantes adultos, en el Reino Unido-Inglaterra/Gales/Irlanda del Norte el $65 \%$ de los estudiantes de títulos profesionales (Foundation Degrees) tiene más de 21 años cuando accede a los estudios, y en Dinamarca el 60\%. Por otro lado, no es de sorprender que Eslovenia también cuente con una mayoría de estudiantes adultos (55\%). De hecho, estos estudiantes adultos son los que estudian a tiempo parcial.

Por otro lado, se les preguntó a los ministerios y a las instituciones si el número de estudiantes desfavorecidos era proporcionalmente mayor en la Educación Superior de ciclo corto o en sus instituciones. Una mayoría de instituciones consideró que la proporción de estudiantes desfavorecidos es superior a la media. Esto podría estar relacionado con el hecho de que, aunque no haya datos disponibles a nivel nacional, las propias instituciones poseen más información sobre la condición social de sus estudiantes.

La cooperación con la industria y otros interlocutores sociales es muy valorada en la Educación Superior de ciclo corto en el conjunto de Europa. De hecho, en algunos países es obligatoria. Esto se refleja en la participación de la industria en todos los aspectos de la Educación Superior de ciclo corto. Así, los profesores que cuentan con experiencia en el mundo de la industria son valorados muy positivamente en todos los países con este tipo de educación. De manera similar, las prácticas y la formación profesional dual tienen gran importancia. La empleabilidad es otra cuestión que también se tiene muy en cuenta y que se fomenta de diversas formas mediante una estrecha cooperación con la industria. 


\section{DISCUSIÓN Y CONCLUSIONES}

Como se defiende desde EURASHE (2011), el sector de la Educación Superior de ciclo corto constituye un elemento esencial en la cadena del aprendizaje permanente. Se trata claramente del eslabón entre la educación secundaria y la Educación Superior y para muchos estudiantes es el vínculo que les permite acceder a la Educación Superior, incluso sin un título de educación secundaria.

De esta forma, se puede afirmar que la Educación Superior de ciclo corto está contribuyendo a ampliar la participación en la Educación Superior en el entorno europeo, puesto que, aparentemente, hay más estudiantes adultos y a tiempo parcial y, también muy probablemente, más estudiantes desfavorecidos que en otros programas de Educación Superior.

La población a la que están destinados estos programas es cada vez más diversa. El hecho de que la Educación Superior de ciclo corto se imparta en una amplia variedad de escenarios como universidades, centros de educación postobligatoria, escuelas profesionales, centros de educación de adultos, etc., facilita sin duda el acceso a la Educación Superior y, por lo tanto, contribuye a ampliar la participación en la misma, así como su dimensión social.

Por otro lado, acorde a lo planteado en otro estudio de EURASHE (2013), esta Educación Superior de marcado carácter profesional puede jugar un importante rol como intermediario entre el mundo universitario y el laboral, facilitando la comprensión entre la Educación Superior y el mercado de trabajo.

A pesar de todo ello, este sector de la Educación Superior no goza en muchos países del mismo prestigio que los estudios universitarios y, en el mejor de los casos, es considerado como el «hermano menor» de la Universidad. Así, por ejemplo, en nuestro país la oferta formativa en Educación Superior -Ciclos Formativos de Grado Superior (CFGS) y estudios universitarios- ha estado segregada tradicionalmente cuando en realidad, en el actual entramado sociolaboral, ambas opciones están llamadas a encontrarse.

La llamada Educación Superior no universitaria se muestra como un terreno insuficientemente investigado, signado además por un acentuado desconocimiento supranacional. En síntesis, muchos interrogantes se abren en torno a la propia definición, así como la pertinencia del nivel en la cadena de la formación permanente y en la búsqueda de la equidad.

\section{REFERENCIAS BIBLIOGRÁFICAS}

CEDEFOP (2014). Qualifications at level 5: progressing in a career or to higher education. Working paper $n^{\circ} 23$. Luxembourg: Publications Office of the European Union.

Cremonini, L. (2010). Short-Cycle Higher Education: An International Review. Países Bajos: Centro de Estudios de Políticas de Educación Superior (CHEPS).

EURASHE (2013). Profile of professional higher education in Europe. Bruselas: EURASHE.

European Commission/EACEA/Eurydice (2015). The European Higher Education Area in 2015: Bologna Process Implementation Report. Luxembourg: Publications Office of the European Union.

González Silva, H. J. (2006). Instituciones de Educación Superior «No Universitaria». En Informe sobre la Educación Superior en América Latina y el Caribe, 2000-2005. La metamorfosis de la Educación Superior. Venezuela: Editorial Metrópolis.

Jacinto, C. (coord.) (2013). Incluir a los jóvenes. Retos para la educación terciaria técnica en América Latina. París: Instituto Internacional de Planeamiento de la Educación (UNESCO).

Kirsch, M. y Beernaert, Y. (2011). Short Cycle Higher Education in Europe Level 5: the Missing Link. Bruselas: EURASHE.

OECD, European Union, UNESCO-UIS (2015). ISCED 2011: Operational Manual Guidelines for classifying national education programmes and related qualifications.

Teichler, U. (2009). Sistemas comparados de Educación Superior en Europa: Marcos conceptuales, resultados empíricos y perspectiva de futuro. Editorial Octaedro, S.L.

UNESCO (2009). Comunicado del 8 de julio de 2009: Conferencia Mundial sobre la Educación Superior - 2009: La nueva dinámica de la Educación Superior y la investigación para el cambio social y el desarrollo. París: UNESCO. 